\title{
Adherencia terapéutica en pacientes anticoagulados con prótesis valvular mecánica
}

\author{
Therapeutic Adherence in anticoagulated patients with a prosthetic mechanical valve \\ Aderência terapêutica em pacientes anticoagulados com próteses valvares cardíacas \\ mecânicas
}

Débora Milena Álvarez-Yañez* Asly Nataly Guevara-García** Yari Paola García-Calderón ***

\begin{abstract}
Autor de correspondencia
* Enfermera. Magister en Práctica pedagógica. Especialista en cuidado de enfermería al paciente crítico. Docente de Universidad Francisco de Paula Santander. Correo:deboramilenaay@ufps. edu.co. (D) https://orcid.org/0000-00024836-9857. Cúcuta, Colombia.

** Enfermera. Universidad Francisco de Paula Santander. Correo: aslynatalygg@ufps.edu. co. (iD) https://orcid.org/0000-0003-24000761. Cúcuta, Colombia.

*** Enfermera. Universidad Francisco de Paula Santander. Correo ypgc ajgc@hotmail. com. (iD https://orcid.org/0000-0002-28926871. Cúcuta, Colombia.
\end{abstract}

\section{Resumen}

Objetivo: Describir el nivel de adherencia terapéutica de los pacientes con prótesis valvular mecánica que reciben anticoagulación oral en una institución de IV nivel. Materiales y Métodos: Estudio cuantitativo descriptivo y de corte transversal, realizado durante el segundo semestre de los años 2016 y 2017. La muestra estuvo representada por 89 pacientes con reemplazo valvular mecánico que fueron sometidos a cirugía cardiaca en una institución de IV nivel durante los años 2011 al 2016. Se utilizó el cuestionario como "Instrumento para evaluar los factores que influyen en la adherencia a tratamientos farmacológicos y no farmacológicos en pacientes con factores de riesgo de enfermedad cardiovascular versión 3", elaborado por Bonilla y de Reales en el 2006. El cuestionario estuvo conformado por 53 ítems y 4 dimensiones: factores socioeconómicos, y los relacionados con el proveedor, la terapia y el paciente. El instrumento tiene un coeficiente Alfa de Cronbach de 0.82 Resultados: El $90 \%$ de los pacientes presentaron un nivel de adherencia bueno; los factores que intervinieron a favor son aquellos relacionados con el sistema y el equipo de salud; la dimensión vinculada a factores socioeconómicos influyó negativamente en la adherencia al tratamiento. Conclusión: Existe una buena adherencia terapéutica en los pacientes encuestados; de igual manera se deduce que es necesario promover acciones interdisciplinarias en los programas de seguimiento a pacientes anticoagulados, enfocadas en los factores que influyen en la adherencia terapéutica.

Palabras Clave: Anticoagulantes, prótesis valvulares cardiacas, enfermería cardiovascular, cumplimiento de la medicación, rol de la enfermera.

\section{Abstract}

Objective: To describe the level of therapeutic adherence of patients with a prosthetic mechanical valve who receive oral anticoagulants in an institution of IV level. (https://creativecommons.org/

licenses $/$ by $/ 4.0 /$ ) (c) (1)

DOI: http://dx.doi.org/10.22463/17949831.1400 
Materials and Methods: Descriptive, quantitative and cross-sectional study performed during the second semester of 2016 and 2017. The sample was composed by 89 patients with prosthetic mechanical valves who were subjected to cardiac surgery in an institution of IV level from 2011 to 2016. The survey used was "Instrument to evaluate the factors that influence the adherence to pharmacological and nonpharmacological treatments in patients with risk factors of cardiovascular disease, third version", elaborated by Bonilla and de Reales in 2006. The survey was composed of 53 items and 4 dimensions: socioeconomic factors, and factors related to the provider, the therapy and the patient. The instrument has a Cronbach Alpha coefficient of 0.82 . Results: 90\% of the patients showed a good level of adherence; the factors that intervened in favor, were those related with the system and the health equipment; the dimension linked to socioeconomic factors intervened negatively in the adherence to the treatment. Conclusion: Good therapeutic adherence exists in the surveyed patients; also, it is assumed that its necessary to promote interdisciplinary actions in the monitoring programs for anticoagulated patients, such actions should be focused on factors that influence in the therapeutic adherence.

Keywords: Anticoagulants, prosthetic heart valves, cardiovascular nursing, adherence to medication, nursing role.

\section{Resumo}

Objetivo: Descrever o nível de aderência terapêutica dos pacientes com prótese valvar cardíaca mecânica que receberam anticoagulação oral numa instituição de IV nível. Materiais e Métodos: Estudo quantitativo descritivo e de corte transversal, realizado durante o segundo semestre dos anos 2016 e 2017. A amostra esteve representada por 89 pacientes com substituição valvar mecânica que foram submetidos a cirurgia cardíaca numa instituição de IV nível durante os anos 2011 a 2016. Utilizou-se o questionário como "Instrumento para avaliar os fatores que influem na aderência a tratamentos farmacológicos e não farmacológicos em pacientes com fatores de risco de doença cardiovascular versão 3", elaborado por Bonilla e de Reales no ano de 2006. O questionário esteve conformado por 53 itens e 4 dimensões: fatores socioeconómicos, e os relacionados como o fornecedor, a terapia e o paciente. O instrumento tem um coeficiente Alfa de Cronbach de 0,82 Resultados: O 90 \% dos pacientes apresentaram um nível de aderência bom; os fatores que interviram a favor foram aqueles relacionados como sistema e a equipe de saúde; a dimensão vinculada a fatores socioeconómicos influiu negativamente na aderência ao tratamento. Conclusões: Existe uma boa aderência terapêutica nos pacientes pesquisados; de igual maneira se deduze que é necessário promover ações interdisciplinares nos programas de seguimento a pacientes anticoagulados, focadas nos fatores que influem na aderência terapêutica.

Palavras-chave: Anticoagulantes, cumprimento da medicação, enfermagem cardiovascular, papel da enfermeira, prótese valvar cardíaca
ISSN-PRINT

1794-9831

E-ISSN 2322-7028

Vol. 15 No. 2

Jul - Dic 2018

Cúcuta, Colombia

\section{Introducción}

Las enfermedades cardiovasculares son la principal causa de discapacidad y muerte en países desarrollados y en vía de desarrollo; afectan en mayor medida a países de ingresos bajos y medianos; más del $80 \%$ de las defunciones se producen en esos países. Según la OMS (Organización Mundial de la Salud) se estima que para el año 2030, casi 23,6 millones de personas morirán por alguna enfermedad cardiovascular, principalmente por cardiopatías y accidentes cerebrovasculares (1). Es necesario recalcar que las valvulopatias son una de las patologías más frecuentes, siendo la válvula aórtica y mitral las más afectadas. Entre las principales causas de esta afección se destacan: la fiebre reumática, degeneración mixomatosa, degeneración cálcica, endocarditis, los ataques y fallas cardiacas, entre otras (2).

Como resultado de estas lesiones valvulares graves o potencialmente mortales, se hace necesario reparar o reemplazar las válvulas cardíacas afectadas. En este tipo de procedimiento se pueden usar dos tipos de válvulas (3): biológica o mecánica. Las válvulas biológicas están hechas generalmente de tejido animal o del tejido de un corazón humano donado, en ocasiones es posible usar el tejido del propio paciente en la sustitución 
ISSN-PRINT

1794-9831

E-ISSN 2322-7028

Vol. 15 No. 2

Jul - Dic 2018

Cúcuta, Colombia valvular (4), poseen una vida útil de 12 a 15 años; por su parte, las válvulas mecánicas están fabricadas de materiales tales como el plástico, el carbono o el metal, tienen una vida útil entre los 20 y 30 años (4). En Estados Unidos se realizan alrededor de 106.000 intervenciones de válvulas cardíacas por año (5). A nivel nacional, el Ministerio de salud y la protección social de Colombia reportó 9.436 procedimientos de reemplazo valvular, entre los años 2009-2015 (6). Durante el periodo comprendido entre 2011 y 2016, una Institución de IV nivel en Cúcuta, reportó 1.260 cirugías cardiacas, de las cuales 320 corresponden a reemplazos valvulares, a 155 pacientes se les realizó reemplazo valvular con prótesis mecánica (7).

Generalmente las válvulas mecánicas tienen una vida útil mayor. Sin embargo, con el tiempo, estas prótesis pueden obstruirse, razón por la cual, los pacientes portadores de estas válvulas deben tomar anticoagulantes diariamente por el resto de su vida (3). Debido al tratamiento terapéutico y los riesgos a los que se ven expuestos estos pacientes, se hace necesario el seguimiento periódico del estado de salud, en relación al tratamiento e indicaciones dadas por el profesional de enfermería. De ahí, que al profesional de la enfermería se le considere como uno de los pilares más importantes, con su actuación oportuna a favor de la adherencia y el compromiso de estos pacientes con su salud, lo que reduce el riesgo de sufrir complicaciones; asimismo, deben reflejar el bienestar y la calidad de vida de los mismos.

Según la Organización Mundial de la Salud, la adherencia terapéutica es definida como el grado en que el comportamiento de una persona influye en la ingesta de la medicación y el cumplimiento del régimen alimentario establecido, también le permite realizar cambios en el estilo de vida de acuerdo a las recomendaciones proporcionadas por el equipo de salud (8). Se destaca que una buena adherencia se puntualiza como una ingesta del $80 \%$ de la dosis prescrita (9). La adherencia al tratamiento farmacológico y no farmacológico es un aspecto clave en el seguimiento del cuidado de la salud, ya que involucra el compromiso del paciente y el actuar del profesional de enfermería en la oferta del cuidado oportuno e íntegro, del desequilibrio de los factores socioeconómicos, y de los factores relacionados con el proveedor; es decir, de sistema y el equipo de salud, así como los relacionados con la terapia y finalmente los que tienen que ver propiamente con el paciente, que influyen en la adherencia terapéutica aumentando el riesgo de complicaciones en la salud del paciente. Los factores que influyen en la adherencia requieren de una relación terapéutica entre los pacientes y el personal de salud para obtener un régimen de anticoagulación oral con valores óptimos; es fundamental que el paciente comprenda la importancia del tratamiento y el cumplimiento de las recomendaciones en cuanto a alimentación, actividad física, apoyo familiar y controles médicos. La adherencia terapéutica es un factor muy susceptible a ser modificable, (10) por tanto, es indispensable que el equipo de salud identifique los factores que intervienen en la adherencia terapéutica como son: los socioeconómicos, los relacionados con el proveedor respecto al sistema y al equipo de salud, los relacionados con la terapia y los vinculados propiamente con el paciente.

Los Factores socioeconómicos influyen de manera significativa, por este motivo se debe identificar las posibilidades económicas para dirigir los recursos hacia la satisfacción de las necesidades básicas como alimentación, vivienda, educación y salud, lo cual favorece la dispensación puntual de los medicamentos por la institución (11).

Respecto a los Factores relacionados con el proveedor, que tienen que ver con el equipo de salud interdisciplinario, desempeñan un aspecto clave para la adherencia terapéutica, sus acciones están enfocadas en lograr que el cuidado de la salud sea seguro (12). De hecho, las buenas relaciones entre los pacientes y sus prestadores de asistencia sanitaria son imperativas para la buena adherencia (13).

Los Factores relacionados con la terapia se asocian al cumplimiento de las citas por parte del paciente, así como al cumplimiento de los diversos tratamientos. En ese caso, se hace énfasis en la posibilidad de seguir las indicaciones relacionadas con los medicamentos, las dietas, los ejercicios y hábitos saludables en general (13). La medicación pertinente y eficaz que genera mejoría percibida por el paciente es el factor que más contribuye a la adherencia (14).

\section{Los Factores relacionados con el paciente} comprenden los recursos, el conocimiento, las actitudes, las creencias, las percepciones y las expectativas del paciente. El conocimiento y las creencias del paciente acerca de su enfermedad, la motivación para tratarla, 
la confianza en su capacidad para involucrarse en los comportamientos terapéuticos y las expectativas con respecto al resultado del tratamiento interactúan de un modo todavía no plenamente comprendido para influir sobre el comportamiento de adherencia (13).

Por consiguiente, es fundamental la responsabilidad del paciente respecto al cuidado de su salud. Del mismo modo, es el profesional de la enfermería quien brinda una atención centrada en las necesidades del paciente, identificando el nivel de adherencia al tratamiento y los factores que intervienen en la misma. Igualmente, para que la educación ofertada al paciente anticoagulado sea eficiente, es necesario que se potencien los conocimientos del paciente y su grupo familiar a fin de que en conjunto se garantice la seguridad del tratamiento, promoviendo de esta manera, una calidad de vida favorable para que el paciente en su desarrollo familiar, laboral y social, tenga en cuenta que la adherencia terapéutica es un factor muy susceptible a ser modificado (15). Es por esto, que "la información, por sí sola, no es una garantía de que el paciente cumpla el tratamiento", razón por la cual es primordial que el profesional de la enfermería refuerce, a través de la educación la modificación de conductas que contribuyan a la adherencia terapéutica (16). En la valoración de la adherencia farmacológica y no farmacológica, es importante que se identifiquen conductas del paciente relacionadas con el cumplimiento de indicaciones terapéuticas para lograr la gestión eficaz de la propia salud. Por tanto, en la interacción con el paciente anticoagulado la enseñanza debe enfocarse en el cumplimiento del tratamiento para evitar la no adherencia terapéutica (17).

\section{Materiales y Métodos}

Se realizó un estudio cuantitativo, descriptivo y de corte transversal, ejecutado en una Institución de IV nivel en Cúcuta, que reportó 1.260 cirugías cardiacas durante los años 2011 a 2016. La población corresponde a 155 pacientes con reemplazo valvular con prótesis mecánica. En esta investigación participaron ochenta y cinco (85) pacientes, utilizando el muestreo no probabilístico por conveniencia. Las limitantes en la recolección de la información estuvieron relacionadas con el fallecimiento del paciente (11 pacientes), cambio de número telefónico ( 35 pacientes) y decisión de no participar en el estudio (20 pacientes).

Los criterios de inclusión fueron: ser paciente del programa de cirugía cardiaca de una institución de IV nivel de la ciudad de Cúcuta, ser portador de prótesis valvular mecánica que recibe anticoagulación oral, haber sido operado en el periodo de tiempo comprendido entre el 2011 y el 2016, ser mayor de 18 años con competencias de lectura y escritura y que deseara participar en el estudio. La técnica de análisis de datos y sistematización de la información se desarrolló a través del programa SPSS versión 24; asimismo, se realizó el análisis descriptivo mediante distribución de frecuencias y porcentual para las variables cualitativas. Se utilizaron medidas de tendencia central para las variables cuantitativas. Los datos son presentados en tablas y gráficas de barra.

Para la recolección de la información se utilizó el "Instrumento para evaluar los factores que influyen en la adherencia a tratamientos farmacológicos $y$ no farmacológicos en pacientes con factores de riesgo de enfermedad cardiovascular versión 3", elaborado por Bonilla y de Rales en el 2006. Con respecto a la confiabilidad y validez se hace referencia a Acelas, quien ejecutó una investigación en el 2008 con el fin de realizar la prueba de confiabilidad del instrumento versión 3, de 53 ítems a través del programa stata 10, encontrando un Alfa de Cronbach de 0.851 , lo cual indica homogeneidad en el instrumento (18).

De igual manera, Ortiz (18) elaboró una investigación con el objetivo de determinar la validez del constructo y confiabilidad del mismo, versión con 53 ítems. Este estudio se realizó con 485 sujetos en la ciudad de Bogotá; se determinó la validez del constructo por medio del análisis factorial de tipo exploratorio con carga factorial de 0.4 para medirlo con el programa SPSS de Windows; se determinó la confiabilidad a través del coeficiente Alfa de Cronbach arrojando un resultado de 0.82 . El instrumento está conformado por 53 ítems y 4 dimensiones: factores socioeconómicos, factores relacionados con el proveedor: sistema y equipo de salud, factores relacionados con la terapia $\mathrm{y}$ factores relacionados con el paciente; tiene un puntaje mínimo 0 puntos y un máximo 106 puntos. Para evaluar las respectivas dimensiones se tuvo en cuenta que puntajes entre el 80 y $100 \%$ referencian una buena adherencia; entre el 60 y $79 \%$ determina riesgo de no adherencia y menos del 59\% muestran una baja adherencia al tratamiento.

Se respetaron los principios éticos de la investigación $\overline{\text { E-ISSN 2322-7028 }}$

Vol. 15 No. 2

Jul - Dic 2018

Cúcuta, Colombia 
ISSN-PRINT

1794-9831

E-ISSN 2322-7028

Vol. 15 No. 2

Jul - Dic 2018

Cúcuta, Colombia en Colombia, resolución 008430 de 1993 (19), utilizando dos tipos de consentimiento informado: uno de forma escrita para aquellos instrumentos de aplicación directa y un consentimiento verbal para la aplicación indirecta del instrumento; así mismo se garantizó la total privacidad de la información.

\section{Objetivos}

\section{Objetivo General}

Describir el nivel de adherencia terapéutica de los pacientes con prótesis valvular mecánica que reciben anticoagulación oral en una institución de IV nivel.

\section{Objetivos Específicos}

- Caracterizar socio-demográficamente la población objeto de estudio.

- Identificar el nivel de adherencia según la dimensión I: factores socioeconómicos en la población objeto de estudio.

- Determinar el nivel de adherencia según la dimensión II: Factores relacionados con el proveedor: Sistema y equipo de salud en la población objeto de estudio.

- Establecer el nivel de adherencia según la dimensión III: Factores relacionados con la terapia en la población objeto de estudio.

- Describir el nivel de adherencia según la dimensión IV: Factores relacionados con el paciente en la población objeto de estudio.

\section{Resultados}

\section{Caracterización Sociodemográfica}

La mayoría de los participantes eran mayores de 50 años siendo el rango entre 56-60 el de mayor frecuencia $(31.46 \%)$. En cuanto al género predominó el género masculino (52.8\%); el nivel educativo que prevaleció fue primaria $(59,6 \%)$, seguido por secundaria $(24.7$ $\%)$ y analfabetismo (7.9 \%). El estado civil que predominó fue el de ser casado (44.9\%); la mayoría se encontraba entre los estratos $1(37.1 \%)$ y $2(40.4 \%)$; y en promedio cada paciente lleva en tratamiento $2.8 \pm$ 1.8 años; no obstante participaron pacientes que tenían la prótesis desde hacía 5 años y otros con menos de un año (Ver tabla 1).

Tabla 1. Caracterización sociodemográfica

\begin{tabular}{|c|c|c|c|}
\hline Variable & Riesgo de no adherencia & Buena adherencia & $\mathrm{n}(\%)$ \\
\hline \multicolumn{4}{|l|}{ Edad } \\
\hline $21-40$ & 1 & 7 & $8(9 \%)$ \\
\hline $41-60$ & 7 & 48 & $55(61.78 \%)$ \\
\hline Mayores de 60 & 1 & 25 & $26(29.21 \%)$ \\
\hline \multicolumn{4}{|l|}{ Sexo } \\
\hline Masculino & $2(4,3 \%)$ & $45(95,7 \%)$ & $47(\mathbf{5 2 , 8 \%})$ \\
\hline Femenino & $7(16,7 \%)$ & $35(83,3 \%)$ & $42(47,2 \%)$ \\
\hline \multicolumn{4}{|l|}{ Escolaridad } \\
\hline Ninguna & $2(28,6 \%)$ & $5(71,4 \%)$ & $7(7,9 \%)$ \\
\hline Primaria & $4(7,5 \%)$ & $49(92,5 \%)$ & $53(59,6 \%)$ \\
\hline Secundaria & $2(9,1 \%)$ & $20(90,9 \%)$ & $22(24,7 \%)$ \\
\hline Técnico & $1(33,3 \%)$ & $2(66,7 \%)$ & $3(3,4 \%)$ \\
\hline Universidad & $0(0,0 \%)$ & $4(100,0 \%)$ & $4(4,5 \%)$ \\
\hline \multicolumn{4}{|c|}{ Estrato socioeconómico } \\
\hline $1-2$ & $9(13 \%)$ & $60(87 \%)$ & $69(77.5 \%)$ \\
\hline $3-4$ & $0(0 \%)$ & $20(0 \%)$ & $20(\mathbf{2 2 . 5 \%})$ \\
\hline \multicolumn{4}{|l|}{ Estado civil } \\
\hline Soltero(a) & $0(0,0 \%)$ & $17(100,0 \%)$ & $17(\mathbf{1 9}, \mathbf{1} \%)$ \\
\hline Unión Libre & $4(17,4 \%)$ & $19(82,6 \%)$ & $23(\mathbf{2 5 , 8 \% )}$ \\
\hline Casado(a) & $4(10,0 \%)$ & $36(90,0 \%)$ & $40(44,9 \%)$ \\
\hline Separado(a) & $0(0,0 \%)$ & $2(100,0 \%)$ & $2(2,2 \%)$ \\
\hline Viudo(a) & $1(14,3 \%)$ & $6(85,7 \%)$ & $7(7,9 \%)$ \\
\hline
\end{tabular}

Fuente: Datos del estudio 
Al establecer la posible asociación entre el nivel de adherencia al programa y los aspectos sociodemográficos de los pacientes, se logró observar respecto a la edad, que la presencia del riesgo de no adherencia predomina en pacientes que superan los 40 años, es decir, que existe una leve tendencia que a mayor edad mayor riesgo de no adherencia (Ver tabla 1).

En cuanto al género, el riesgo de no adherencia al tratamiento es mayor en mujeres ( $16.7 \%)$, comparada con los hombres (4.3\%); los datos no permiten concluir que exista una diferencia significativa $(\mathrm{p}=0.08)($ Ver tabla 1$)$.

La mayor parte de pacientes con riesgo de no adherencia al tratamiento son personas con baja instrucción escolar; sin embargo, la presencia del riesgo se evidencia en los demás niveles educativos, por lo cual no se puede afirmar que existe asociación estadísticamente significativa respecto a la escolaridad $(\mathrm{p}=0.66)($ Ver tabla 1$)$.

Por otra parte, existe asociación estadísticamente significativa entre el estrato socioeconómico de los pacientes y el nivel de adherencia al tratamiento ( $\mathrm{p}$ $<0.01$ ). El riesgo de no adherencia al tratamiento predomina en pacientes pertenecientes a los estratos
1 y 2 , pero es independiente al estado civil de los pacientes $(\mathrm{p}=0.46)$; no obstante, se observó que la presencia de este riesgo es más frecuente en pacientes en unión libre y personas casadas (Ver tabla 1).

Con respecto a la relación existente entre el tiempo terapéutico y el nivel de adherencia al tratamiento de los pacientes encuestados se evidenció, la existencia de una correlación directa entre el tiempo del tratamiento y el nivel de adherencia al mismo, por lo cual se puede concluir que poseen una relación estadísticamente significativa, a mayor tiempo terapéutico mayor adherencia. $(\mathrm{p}<0.01)$.

\section{Adherencia al tratamiento general}

A nivel general, se identificó que el $90 \%$ de los pacientes valorados manifiesta buena adherencia al tratamiento; mientras que el $10 \%$ se encuentra en riesgo de no adherencia. También se evidenció que la dimensión de factores socioeconómicos influyó negativamente en la adherencia, contrario a la dimensión relacionada con el sistema y equipo de salud que tuvo mayor influencia positiva en la adherencia terapéutica (Ver figura 1).

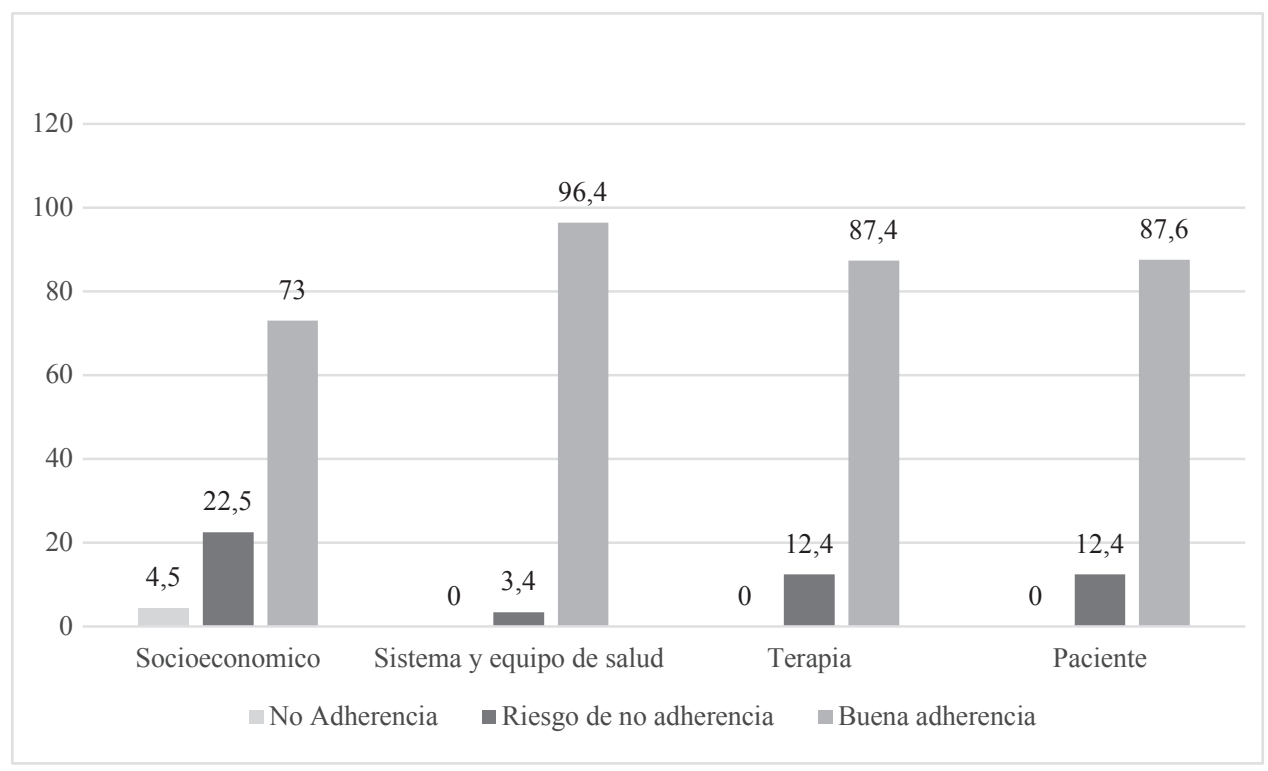

Figura 1: Nivel de adherencia al tratamiento por dimensiones

Fuente: Datos del estudio 
ISSN-PRINT

1794-9831

E-ISSN 2322-7028

Vol. 15 No. 2

Jul - Dic 2018

Cúcuta, Colombia

\section{Dimensión I: Factores socioeconómicos.}

En cuanto a los factores relacionados con los aspectos socioeconómicos, se evidenció que el $73 \%$ de la población posee una buena adherencia al tratamiento (ver figura 1), siendo el ítem con menor puntuación promedio el poder cumplir con los cambios en la dieta debido al alto costo de los alimentos recomendados, seguido del poder costearse los medicamentos, lo cual demuestra que el factor económico es una barrera para la adecuada adherencia al tratamiento. Por otra parte, los participantes manifiestan que a pesar de las dificultades y costos del medicamento, vale la pena conseguirlo y seguir las recomendaciones para el beneficio de la salud (Ver tabla 2).

Tabla 2. Factores socioeconómicos

\begin{tabular}{|c|c|c|}
\hline Factores socioeconómicos & Promedio & $\begin{array}{l}\text { Desviación } \\
\text { Estándar }\end{array}$ \\
\hline $\begin{array}{l}\text { 1. Tiene disponibilidad económica su familia para atender las necesidades básicas: } \\
\text { (alimentación, salud, vivienda, educación.) }\end{array}$ & 1,46 & 0,71 \\
\hline 2. Puede costearse los medicamentos. & 1,10 & 0,81 \\
\hline 3. Cuenta con los recursos económicos para trasladarse al lugar de la consulta. & 1,64 & 0,61 \\
\hline 4. Cuenta con permisos laborales para asistir a sus citas. & 1,92 & 0,34 \\
\hline $\begin{array}{l}\text { 5. Reconoce que a pesar de los costos para conseguir los medicamentos y seguir } \\
\text { recomendaciones vale la pena hacerlo. }\end{array}$ & 2,00 & 0,00 \\
\hline $\begin{array}{l}\text { 6. Los cambios en la dieta, se le dificultan debido al alto costo de los alimentos } \\
\text { recomendados }\end{array}$ & 0,96 & 0,85 \\
\hline 7. Puede leer la información escrita sobre el manejo de su enfermedad. & 1,44 & 0,81 \\
\hline 8. Cuenta con el apoyo de su familia o personas allegadas para cumplir su tratamiento. & 1,91 & 0,36 \\
\hline $\begin{array}{l}\text { 9. Las relaciones entre los miembros de la familia que viven con usted lo desaniman para } \\
\text { seguir los tratamientos. }\end{array}$ & 1,90 & 0,40 \\
\hline $\begin{array}{l}\text { 10. Las diversas ocupaciones que tiene dentro y fuera del hogar le dificultan seguir el } \\
\text { tratamiento. }\end{array}$ & 1,90 & 0,37 \\
\hline $\begin{array}{l}\text { 11. El contacto con otras personas o grupos que están tratando de mejorar su salud le sirven } \\
\text { de ejemplo. }\end{array}$ & 1,83 & 0,46 \\
\hline 12. Los que le ayudan saben qué tan grave es la enfermedad y su tratamiento. & 1,91 & 0,36 \\
\hline $\begin{array}{l}\text { 13. Las distancias de su casa o trabajo a los consultorios le dificultan el cumplimiento de sus } \\
\text { citas. }\end{array}$ & 1,67 & 0,62 \\
\hline $\begin{array}{l}\text { 14. Comparte con otras personas sus preocupaciones y estos lo animan a seguir su } \\
\text { tratamiento. }\end{array}$ & 1,42 & 0,81 \\
\hline
\end{tabular}

Fuente: Datos del estudio

\section{Dimensión II: Factores relacionados con el proveedor: Sistema y equipo de salud.}

El $96.6 \%$ de los pacientes manifiestan buena adherencia terapéutica (ver figura 1), aunque se encontró que el ítem con menor promedio es "en el caso que usted fallará en su tratamiento su médico y enfermera entenderían sus motivos"; mientras que los ítems con mayor promedio fueron: "las personas que lo atienden responden sus inquietudes y dificultades con respecto a su tratamiento"; "el médico y la enfermera le dan explicaciones con palabras que su familia o usted entienden"; y, "el trato que recibe del personal médico y los demás es el que usted espera" lo cual evidencia el compromiso del personal de enfermería para brindar una atención de calidad al paciente favoreciendo su estado de salud (Ver tabla $3)$. 
Tabla 3. Factores relacionados con el proveedor: sistema y equipo de salud

\begin{tabular}{|c|c|c|}
\hline Factores relacionados con el proveedor & Promedio & $\begin{array}{l}\text { Desviación } \\
\text { Estándar }\end{array}$ \\
\hline 15. El trato del médico y las enfermeras lo anima a volver a sus controles. & 1,98 & 0,15 \\
\hline 16. El personal de enfermería le enseña y da recomendaciones escritas sobre sus tratamientos. & 1,96 & 0,26 \\
\hline 17. La información verbal de parte del médico es detallada y precisa. & 1,96 & 0,21 \\
\hline $\begin{array}{l}\text { 18. Tiene dudas acerca de la manera de tomar sus medicamentos, en cuanto a la cantidad, los horarios y } \\
\text { la relación con las comidas. }\end{array}$ & 1,93 & 0,29 \\
\hline 19. La institución a la que consulta muestra desorganización en la atención que le brinda. & 1,93 & 0,33 \\
\hline 20. Las personas que lo atienden se ven demasiado ocupadas para escucharlo por mucho tiempo. & 1,97 & 0,24 \\
\hline $\begin{array}{l}\text { 21. Las personas que lo atienden responden sus inquietudes y dificultades con respecto a su } \\
\text { tratamiento. }\end{array}$ & 1,99 & 0,11 \\
\hline 22. Se da cuenta que su médico controla si está siguiendo el tratamiento por las preguntas que le hace. & 1,89 & 0,35 \\
\hline 23. Recibe información sobre los beneficios de los medicamentos ordenados por su médico. & 1,89 & 0,44 \\
\hline $\begin{array}{l}\text { 24. Recibe orientación sobre la forma de ajustar los horarios de los medicamentos de acuerdo a sus } \\
\text { actividades diarias. }\end{array}$ & 1,92 & 0,31 \\
\hline 25. En el caso que usted fallará en su tratamiento su médico y enfermera entenderían sus motivos. & 1,49 & 0,68 \\
\hline 26. Conoce por medio escrito que señala fecha, horario y lugar del próximo control. & 1,97 & 0,18 \\
\hline 27. El médico y la enfermera le dan explicaciones con palabras que su familia o usted entienden. & 1,99 & 0,11 \\
\hline 28. Cuando le cambian el médico que lo atiende, esto lo confunde. & 1,69 & 0,65 \\
\hline 29. El cambio frecuente de medicamentos lo confunde. & 1,82 & 0,53 \\
\hline 30. Siente que no recibe atención de salud con la misma calidad que los demás. & 1,96 & 0,26 \\
\hline $\begin{array}{l}\text { 31. El médico y la enfermera le han explicado qué resultados va a tener en su salud con el tratamiento } \\
\text { que se le está dando. }\end{array}$ & 1,96 & 0,30 \\
\hline 32. El trato que recibe del personal médico y los demás es el que usted espera. & 1,99 & 0,11 \\
\hline $\begin{array}{l}\text { 33. La institución de salud le da oportunidades de aprender a reconocer los riesgos que tiene y como } \\
\text { modificarlos. }\end{array}$ & 1,87 & 0,46 \\
\hline 34. Conoce los riesgos que llevan a una persona a sufrir enfermedad cardiovascular. & 1,62 & 0,76 \\
\hline 35. Puede conseguir sus medicamentos de acuerdo al tipo de afiliación a la que pertenece & 1,75 & 0,55 \\
\hline
\end{tabular}

Fuente: Datos del estudio.

Dimensión III: Factores relacionados con la terapia.

El $87.6 \%$ de los pacientes demostró buena adherencia al tratamiento en esta dimensión (ver figura 1). Con respecto al ítem "cree al igual que su familia que todo este tratamiento y cambios en sus costumbres son contrarios a su fe y sus valores" fue el de menor promedio a diferencia del ítem "piensa que algunos de los medicamentos, le crean dependencia por eso no lo toma". Los participantes reconocen que el tratamiento no le crea ningún tipo de adicción o dependencia y es solo para beneficio propio; también están de acuerdo en que vale la pena cumplir el tratamiento y las recomendaciones para mejorar su salud (Ver tabla 4). 
ISSN-PRINT

1794-9831

E-ISSN 2322-7028

Vol. 15 No. 2

Jul - Dic 2018

Cúcuta, Colombia

Tabla 4. Factores relacionados con la terapia

\begin{tabular}{lcc}
\hline \multicolumn{1}{c}{ Factores relacionados con la terapia } & Promedio & $\begin{array}{c}\text { Desviación } \\
\text { Estándar }\end{array}$ \\
\hline 36. Tiene forma de distinguir los diferentes medicamentos para no confundirlos. & 1,97 & 0,24 \\
37. Cuando mejoran sus síntomas, usted suspende el tratamiento. & 1,94 & 0,28 \\
38. Anteriormente ha presentado dificultades para cumplir su tratamiento. & 1,85 & 0,39 \\
39. Piensa que algunos de los medicamentos, le crean dependencia por eso no los toma. & 2,00 & 0,00 \\
40. Está convencido que el tratamiento es beneficioso y por eso sigue tomándolo. & 1,98 & 0,21 \\
41. Cree al igual que su familia que todo este tratamiento y cambios en sus costumbres son & 0,07 & 0,36 \\
contrarios a su fe y sus valores. & 2,00 & 0,00 \\
42. Reconoce que vale la pena cumplir el tratamiento y las recomendaciones para mejorar su salud. & 1,07 & 0,89 \\
43. Cree que hay costumbres sobre alimentos y ejercicios difíciles de cambiar. & 1,92 & 0,38 \\
\hline 44. Cree conveniente para su salud controlar el peso, mediante la dieta y el ejercicio. & 1,92 & 0,34 \\
\hline 45. Los medicamentos que toma actualmente le alivian los síntomas. &
\end{tabular}

Fuente: Datos del estudio.

\section{Dimensión IV: Factores relacionados con el paciente}

El $87.6 \%$ de los pacientes manifestaron buena adherencia al tratamiento (Ver figura 1). En menor promedio los participantes manifestaron que en algún momento sintieron angustia y desánimo por las dificultades para manejar su enfermedad; sin embargo, la totalidad de los encuestados reconoce la importancia de la continuidad del tratamiento (Ver tabla 5).

Tabla 5. Factores relacionados con el paciente

\begin{tabular}{lcc}
\hline \multicolumn{1}{c}{ Factores relacionados con el paciente } & Promedio & $\begin{array}{c}\text { Desviación } \\
\text { Estándar }\end{array}$ \\
\hline 46. Su enfermedad limita sus oportunidades de estar con otras personas. & 1,75 & 0,61 \\
$\begin{array}{l}\text { 47. Le parece que el médico y usted coinciden en la esperanza de mejoría con el tratamiento y los } \\
\text { cambios que está haciendo en sus hábitos. }\end{array}$ & 1,93 & 0,33 \\
$\begin{array}{l}\text { 48. Siente rabia con la enfermedad por las incomodidades que le produce. } \\
\text { 49. Se interesa por conocer sobre su condición de salud y la forma de cuidarse. }\end{array}$ & 1,56 & 0,62 \\
\hline 50. Se angustia y se siente desanimado por las dificultades para manejar su enfermedad. & 1,80 & 0,48 \\
\hline $\begin{array}{l}\text { 51. Se siente discriminado o alejado de su familia o grupo a causa de los tratamientos y } \\
\text { recomendaciones que tiene que seguir. }\end{array}$ & 1,52 & 0,68 \\
\hline 52. Cree que es importante seguir su tratamiento para mejorar su salud. & 1,98 & 0,15 \\
\hline 53. Cree que es usted es el responsable de seguir el cuidado de su salud. & 2,00 & 0,00 \\
\hline
\end{tabular}

Fuente: Datos del estudio.

\section{Discusión}

El análisis de los datos sociodemográficos determinó que el grupo etario en riesgo de no adherencia son los pacientes que superan los 40 años, lo que difiere con el estudio realizado por Salcedo y Gómez (20), quienes destacan que no existe una conceptualización que permita relacionar fuertemente la idea de que a mayor edad, mayor riesgo de abandono al tratamiento; por su parte Contreras et al. (11) advierten que cuando la edad supera los 75 años y existe una patología concomitante limitante con la tensión arterial, el riesgo de no adherencia es alto. 
En relación al estado civil, se evidenció que la presencia de riesgo en no adherirse al tratamiento es más frecuente en pacientes en unión libre y personas casadas, locual difiere del estudio realizado por Zambrano R et al. (13) donde se evidencia que al recibir apoyo por parte de la familia conlleva de manera positiva a la adherencia terapéutica; así mismo, en el estudio realizado por Contreras et al. (11) se demostró que la falta de redes de apoyo social, condiciones de vida inestable y la disfunción familiar, entre otros, actúa de manera negativa sobre la adherencia al tratamiento.

Del mismo modo, los factores socioeconómicos influyen de manera significativa en la adherencia terapéutica; sin embargo es importante señalar que las posibilidades económicas influyen en la satisfacción de las necesidades básicas como alimentación, vivienda, educacióny salud, asícomo en la dispensación puntual de los medicamentos al paciente (11). Se evidencia que los pacientes no pueden costear sus medicamentos debido al costo de estos, más cuando este tipo de medicamento es permanente. Al respecto, Ávila et al. (21) refieren que las dificultades económicas constituyen barreras para la adhesión al tratamiento con anticoagulante y que la indisponibilidad de acceso a la medicación es poco valorado por algunos profesionales. No obstante, Ortega y Vargas (22) encontraron que entre el $66.9 \%$ y el $83.1 \%$ de los pacientes siempre reconocen que vale la pena conseguir los medicamentos a pesar de su costo y asistir a la consulta; además, encontraron que los pacientes estaban en capacidad de leer la información escrita sobre el manejo de su enfermedad y contaban con apoyo familiar-social para cumplir su tratamiento

Los factores relacionados con el proveedor: Sistema y equipo de salud influyen en la adherencia terapéutica, sus acciones están enfocadas en lograrque el cuidado de la salud sea seguro. Se encontró una buena adherencia en esta dimensión; en el ítem "recibe información sobre los beneficios de los medicamentos ordenados por su médico" se obtuvo una puntuación de 1.89, similar a la que Veliz et al. (23) quienes hallaron que el $1.5 \%$ de las personas encuestadas siempre siguen las indicaciones entregadas por el equipo de salud. En cuanto a la comunicación que el personal de salud le ofrece a los pacientes, ya sea mediante información, resolviendo sus dudas sobre el tratamiento prescrito, o informándoles sobre qué beneficios le traerá este tratamiento, Huertas et al. (24) mencionan que la constante comunicación con el equipo de salud influye en un alto grado de adherencia. Por otra parte, Ortega y Vargas (22) encontraron que más del 80 $\%$ de participantes siempre "recibían información y explicaciones por parte del médico y de la enfermera con palabras entendibles sobre resultados, beneficios y formas de ajustar los horarios de los medicamentos de acuerdo a sus actividades diarias".

Para Zambrano et al. (13) los factores relacionados con la terapia se asocian al cumplimiento de las citas por parte del paciente, así como a la consecución del tratamiento; también se enfatiza en la necesidad de seguir las indicaciones relacionadas con los medicamentos, las dietas, los ejercicios y hábitos saludables en general. La mayoría de participantes manifestaron tener una buena adherencia en lo relacionado a esta dimensión: respecto al ítem, "anteriormente ha presentado dificultades para cumplir su tratamiento" gran parte de la población manifestó no haber presentado dificultades que interfirieran en el mismo. No obstante, Pérez et al. (25) reseñaron que la mayoría de los pacientes percibieron muchas barreras para poder cumplir con las indicaciones de su médico tratante.

Respecto al ítem "cuando mejoran sus síntomas, usted suspende el tratamiento", en mayor proporción la población manifestó nunca hacerlo, similar a lo que dicen Van et al. (26) al señalar que más de la mitad de la población declaró que no habían perdido una dosis de su medicación durante el último mes. De igual manera, Terechenko et al. (27) refieren que un gran porcentaje no abandonaba la medicación aunque se encontrara bien. En este contexto, la totalidad de la población encuestada reconoció que vale la pena cumplir el tratamiento y las recomendaciones para mejorar su salud. No se debe olvidar, que hay costumbres sobre alimentos y ejercicios que son difíciles de cambiar, aunque gran parte de la población siempre cree conveniente para su salud controlar el peso, mediante la dieta y el ejercicio; parámetros similares a los que indica Romero (28) para quien más de la mitad de los pacientes tenían una buena adherencia al tratamiento y buen comportamiento ante otros regímenes terapéuticos, como la dieta, el ejercicio, entre otros. Por su parte, Terechenko et al. (27) difieren al especificar que la mayor falencia que presenta esta población en relación con la adherencia al tratamiento es la adquisición de hábitos saludables; de igual manera, Freitas et al. (29) demostraron que existe una menor adherencia 
ISSN-PRINT

1794-9831

E-ISSN 2322-7028

Vol. 15 No. 2

Jul - Dic 2018

Cúcuta, Colombia a las medidas relacionadas con la supervisión diaria de peso, la adopción de una dieta baja en sodio y la inclusión de jugo de frutas en la cantidad de ingesta diaria de líquidos. Por otro lado, Beltrán y Leal (30), determinaron que un poco más de la mitad de los pacientes fueron cumplidores, no olvidaban tomar nunca la medicación y no abandonaban el tratamiento cuando se encontraban bien o cuando les sentaba mal.

Teniendo en cuenta, los factores relacionados con el paciente que incluyen los conocimientos, las actitudes, la motivación, los factores emocionalesy las expectativas con respecto al resultado del tratamiento (13), los hallazgos del presente estudio muestran que la mayoría de los pacientes posee una buena adherencia al tratamiento en lo relacionado a esta dimensión; por el contrario, para Salcedo y Gómez (20) la mayoría de la población presenta un riesgo de adherencia bajo en lo relacionado a esta dimensión. Con respecto al ítem "su enfermedad limita sus oportunidades de estar con otras personas" la mayor parte de la población objeto de estudio refirió que la enfermedad no limitaba su integración social;así mismo, frente al ítem "se siente discriminado o alejado de su familia o grupo a causa del tratamiento y recomendaciones que tiene que seguir" en un alto porcentaje manifestaron que tenían el apoyo de su familia durante el proceso de terapia. Resultados semejantes a los que obtuvieron Ortega y Vargas (22), donde en mayor proporción los pacientes manifiestan que contaban con apoyo familiar-social para cumplir su tratamiento. Por otra parte, se evidenció que los pacientes en algún momento han sentido rabia con la enfermedad por las incomodidades que les produce e incluso angustia y desanimo por el manejo de la misma. De modo similar, Huertas et al. (24) manifiestan que entre las posibles causas de la no adherencia, se encuentran los factores psicosociales como depresión y ansiedad, entre otros.

Según Salcedo y Gómez (20) existen factores que influyen en la adherencia terapéutica, como las características socioeconómicas, las relacionadas con la enfermedad, con el tratamiento, con el paciente y el sistema de atención sanitaria que afectan el comportamiento de la persona hacia el seguimiento de la medicación o recomendaciones dadas por el profesional de enfermería. En el estudio, el $90 \%$ de los pacientes valorados manifestaron buena adherencia al tratamiento, restando un mínimo porcentaje que se encuentra en riesgo de no adherencia. Con respecto a los factores que influyen en el nivel de adherencia se encontró un mayor nivel de adherencia en la dimensión II: sistema y equipo de salud; por otra parte, la dimensión que influye mayormente en la no adherencia al tratamiento es la dimensión I: relacionada con factores socioeconómicos. Como se puede observar, estos resultados son semejantes a los hallazgos de Ávila el at. (21), en donde la totalidad de la población se encuentra entre adherencia alta y promedio; igualmente se demostró que las dificultades económicas constituyen barreras para la adecuada adhesión al tratamiento. Por otro lado, Salcedo y Gómez (20), evidenciaron que en mayor proporción los pacientes presentan un riesgo moderado y alto de adherencia al tratamiento presentándose el impacto socioeconómico como el mayor influyente en la no adherencia al mismo.

\section{Conclusiones}

- Esta investigación permitió identificar que los factores socioeconómicos y los relacionados con el proveedor: sistema y equipo de salud, son los más influyentes sobre los pacientes en su relación con la adherencia al tratamiento.

- Los factores socioeconómicos son una barrera para la adecuada adherencia al tratamiento debido al alto costo del tratamiento, a pesar de esto los participantes manifiestan que sin importar las dificultades y costos vale la pena seguir las recomendaciones para el beneficio de la salud. De igual manera se evidenció que los factores relacionados con el proveedor tienen un nivel de adherencia bueno. Del mismo modo, los pacientes reconocen el adecuado servicio prestado por el personal de salud, resaltando el trato humanizado del profesional de enfermería, quien acompaña y aconseja al paciente anticoagulado desde el pos operatorio de cirugía cardiaca, educándolo a él y a su familia sobre los cambios en su estilo de vida que incluyen: alimentación, actividad física, apoyo emocional y terapia farmacológica; también le ofrece recomendaciones generales que le permitan el reconocimiento precoz de complicaciones, el compromiso con la continuidad del tratamiento y sus controles periódicos.

- Se demuestra igualmente, que el $90 \%$ de los 
pacientes tienen un nivel de adherencia bueno, destacando que los factores que intervienen a favor del mismo son aquellos relacionados con el sistema y el equipo de salud, al mostrar su compromiso con el paciente. En definitiva, la enfermería a través del cuidado holístico y humanizado contribuye a que el individuo alcance el empoderamiento de su cuidado para garantizar una mejor calidad de vida en el paciente anticoagulado.

\section{Conflicto de intereses}

Las autoras declaran no tener conflicto de intereses

\section{Referencias Bibliográficas}

1. Organización Mundial de la Salud. Enfermedades cardiovasculares, Datos y Cifras. [Internet]. Ginebra: OMS, 2016. [Consultado el 15 de Noviembre de 2016]. Disponible en: http://www.who.int/ mediacentre/factsheets/fs317/es/

2. Liga Colombiana contra el infarto y la hipertensión. Valvulopatía. [Internet]. 2016 [Citado el 1 de nov de 2016]. Disponible en: http://www.colombiacorazon.com/Valvulopatia.html

3. MedlinePlus. Cirugía de válvulas cardíacas. [Internet]. 2017 [Consultado el 15 de Abril de 2017]. Disponible en: https://medlineplus.gov/spanish/ency/article/002954.htm

4. Fundación Española del Corazón. Valvulopatías, Enfermedades, Pacientes. [Internet]. 2016 [Consultado el 15 de Abril de 2017]. Disponible en: http://www.fundaciondelcorazon.com/informacion-parapacientes/enfermedades-cardiovasculares/valvulopatias.html

5. Centro de Información Cardiovascular, Texas Heart Institute. Reparación o sustitución valvular. [Internet]. 2016 [Consultado el 1 de nov de 2016]. Disponible en: http://www.texasheart.org/HIC/ Topics_Esp/Proced/vsurg_span.cfm

6. Ministerio de Salud y Protección Social de Colombia. Remplazos Valvulares, Registro Individual de Prestación de Servicios - RIPS. Bogotá: SISPRO; 2016.

7. Clínica San José de Cúcuta. Servicio de cardiología. Cúcuta: La Clínica; 2016.

8. Sabaté E. Adherencia a los tratamientos a largo plazo. Pruebas para la acción [Internet]. Washington: Organización Panamericana de la Salud; 2004 [Consultado el 22 de Septiembre de 2017]. Disponible

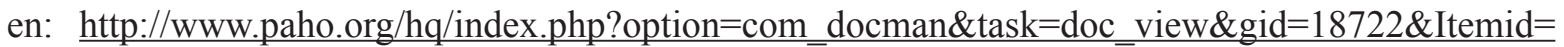
270\&lang=en

9. Ewen S, Rettig V, Mahfoud F, Bo"hm M, Laufs U. Drug adherence in patients taking oral anticoagulation therapy. Clinical Research in Cardiology [Internet]. 2014 [Consultado el 1 de Noviembre de 2016]; 103(3):173-182. Disponible en: http://link.springer.com/article/10.1007\%2Fs00392-013-0616-8

10. Herrera A. Factores que influyen en la adherencia a tratamientos en pacientes con riesgo de enfermedad cardiovascular. Av. enferm. [Internet]. 2008 [consultado el 30 septiembre de 2016]; 26(1):36-42. Disponible en: http://www.revistas.unal.edu.co/index.php/avenferm/article/view/12883/13641

11. Contreras Orozco A, Flórez Torres IE, Herrera Lián A. Un instrumento para evaluar la adherencia: su validez facial y confiabilidad. Av. enferm. [Internet]. 2008 [consultado el 30 septiembre de 2016]; 26(2):35-42. Disponible en: http://www.revistas.unal.edu.co/index.php/avenferm/article/ view/12896/13656

12. Bonilla C. Adherencia y factores que influyen en la adherencia a tratamientos farmacológicos en personas que presentan factores de riesgo de enfermedad cardiovascular: una revisión de literatura. Bogotá: Universidad Nacional de Colombia; 2008.

13. Zambrano R, Duitama F, Posada I, Flórez F. Percepción de la adherencia a tratamientos en pacientes con factores de riesgo cardiovascular. Rev. Fac. Nac. Salud Pública. [Internet]. 2012 [Consultado el 15 de nov de 2016]; 30(2):163-174. Disponible en: http://www.redalyc.org/pdf/120/12023918005.pdf

14. De la Rubia J, Cerda MT. Predictores psicosociales de adherencia a la medicación en pacientes 
ISSN-PRINT

$1794-9831$

E-ISSN 2322-7028

Vol. 15 No. 2

Jul - Dic 2018

Cúcuta, Colombia con diabetes tipo 2. Revista Iberoamericana de Psicología y Salud. [Internet] 2015. [consultado el 30 septiembre de 2016]; 6(1):19-27. Disponible en: http://www.elsevier.es/es-revista-revistaiberoamericana-psicologia-salud-152-articulo-predictores-psicosociales-adherencia-medicacionpacientes-S2171206915700037

15. Organización mundial de la salud (OMS). Formulario Modelo de la OMS 2004. Consejo general a los prescriptores. Adhesión (cumplimiento) al tratamiento farmacológico. [internet]. Ginebra: OMS, 2004. [Consultado el 01 de octubre de 2016]. Disponible en: http://apps.who.int/medicinedocs/es/d/ Js5422s/4.3.html\#Js5422s.4.3

16. Enfermería, clave en la mejora de la adherencia al tratamiento cardiovascular, Notas de Prensa, Noticias de Cardiología, Sociedad Española de Cardiología, [Internet] 2015 [Consultado 02 de Septiembre del 2017] Disponible en: https://secardiologia.es/comunicacion/notas-de-prensa/notas-de-prensasec/6776-enfermeria-clave-en-la-mejora-de-la-adherencia-al-tratamiento-cardiovascular

17. Domínguez S. El papel de la enfermería en la adherencia terapéutica. Elservier, [Internet] 2013 [Consultado el 02 de Septiembre del 2017], Disponible en: https://www.elsevier.es/corp/ generacionelsevier/el-papel-de-la-enfermeria-en-la-adherencia-terapeutica/

18. Ortiz C. Validez y confiabilidad del instrumento Factores que influyen en la adherencia a los tratamientos farmacológicos y no farmacológicos en pacientes con factores de riesgo de enfermedad cardiovascular [Tesis de Maestría] Bogotá: Facultad de Enfermería, Universidad Nacional de Colombia; 2008.

19. Ministerio de Salud y Protección social de Colombia. Resolución 8430 de 1993, octubre 4. Por la cual se establecen las normas científicas, técnicas y administrativas para la investigación en salud. [Internet]. Bogotá: El Ministerio; 1993. [Consultado el 02 noviembre de 2016]. Disponible en: https:// www.minsalud.gov.co/Normatividad_Nuevo/RESOLUCION\%208430\%20DE\%201993.pdf

20. Salcedo A, Gómez A. Grados de riesgo para la adherencia terapéutica en personas con hipertensión arterial. Av. enferm [Internet]. 2014 [Consultado el 15 de nov de 2016]; 32(1): -43. Disponible en: http://www.scielo.org.co/pdf/aven/v32n1/v32n1a04.pdf

21. Ávila W, Aliti B, Feijó F, Rabelo R. Adhesión farmacológica al anticoagulante oral y factores que influyen en la estabilidad del índice de estandarización internacional. Rev. Latino-Am. Enfermagem. [Internet]. 2011 [Consultado el 25 de junio de 2016]. 19(1):1-8. Disponible en: http://www.scielo.br/ pdf/rlae/v19n1/es_04.pdf

22. Ortega Oviedo SI, Vargas Rosero E. Grado de adherencia a tratamientos en personas con riesgo cardiovascular. Av. enferm [Internet]. 2014 [Consultado el 15 de nov de 2016]; 32(1):25-32. Disponible en: http://dx.doi.org/10.15446/av.enferm.v32n1.46032

23. Veliz Rojas L, Mendoza Parra S, Barriga OA. Adherencia terapéutica y control de los factores de riesgo cardiovasculares en usuarios de atención primaria. Enferm. univ [Internet]. 2015 [consultado el 27 de Jun de 2016]; 12(1):3-11. Disponible en: http://www.scielo.org.mx/pdf/eu/v12n1/v12n1a2.pdf

24. Huertas M, Pérez R, Albalate M, Sequera P, Ortega M, Puerta M, et al. Factores psicosociales y adherencia al tratamiento farmacológico en pacientes en hemodiálisis crónica. Nefrología (Madr.) [Internet]. 2014 [Consultado el 15 de nov de 2016]; 34(6): 737-742. Disponible en: https://dx.doi. org/10.3265/Nefrologia.pre2014.Jul.12477

25. Pérez E, Soler Y, Morales L. Adherencia terapéutica y creencias sobre su salud en pacientes hipertensos. MEDISAN [Internet]. 2016 [Citado el 15 Julio de 2016]; 20(1):3-9. Disponible en: http://scielo.sld.cu/ scielo.php?script $=$ sci arttext\&pid $=\mathrm{S} 102930192016000100002$

26. Van Damme S, Van Deyk K, Budts W, Verhamme P, Moons P. Patient knowledge of and adherence to oral anticoagulation therapy after mechanical heart-valve replacement for congenital or acquired valve defects. Heart \& Lung: The Journal of Acute and Critical Care [Internet]. 2011 [Consultado el 15 de nov de 2016]; 40(2):139-146. Disponible en: https://doi.org/10.1016/j.hrtlng.2009.11.005

27. Terechenko N, Baute A, Zamonsky J. Adherencia al tratamiento en pacientes con Diagnóstico de 
Diabetes Mellitus Tipo II. Rev. Biomedicina. [Internet]. 2015 [Consultado el 27 de junio de 2016]; 10(1):20-33. Disponible en: http://www.um.edu.uy/docs/diabetes.pdf

28. Romero A. Adherencia terapéutica de los pacientes en tratamiento anticoagulante oral (warfarina). Enferm Clin. [Internet]. 2013 [Consultado el 30 de junio de 2016]; 23(6):298-299; Disponible en: https://dx.doi.org/10.1016/j.enfcli.2013.09.005

29. Freitas A, Dantas A, Malta M, Silva C, Gandia T, da Fé A, Rejane E. La adherencia al tratamiento de pacientes con insuficiencia cardiaca acompañados de enfermeras en dos clínicas especializadas. Rev. Latino-Am. Enfermagem [Internet]. 2015 [Consultado el 25 de junio de 2016]; 23(5):888-94. Disponible en: https://dx.doi.org/10.1590/0104-1169.0268.2628

30. Beltran A, Leal M. Factores relacionados con la adherencia al tratamiento farmacológico en pacientes con diagnóstico de depresión en una institución de salud de Bogotá. Bogotá: Pontificia Universidad Javeriana; 2009. Disponible en: http://www.javeriana.edu.co/biblos/tesis/enfermeria/2009/ DEFINITIVA/tesis07.pdf
1794-9831

E-ISSN 2322-7028

Vol. 15 No. 2

Jul - Dic 2018

Cúcuta, Colombia 